\title{
ON CERTAIN MEAN VALUES OF THE DOUBLE ZETA-FUNCTION
}

\author{
SOICHI IKEDA, KANEAKI MATSUOKA, \\ AND YOSHIKAZU NAGATA
}

\begin{abstract}
In this article we discuss three types of mean values of the Euler double zeta-function. To get the results, we introduce three approximate formulas for this function.
\end{abstract}

\section{$\S 1$. Introduction}

Let $s_{1}=\sigma_{1}+i t_{1}$, and let $s_{2}=\sigma_{2}+i t_{2}$, with $\sigma_{1}, \sigma_{2}, t_{1}, t_{2} \in \mathbb{R}$. The Euler double zeta-function is defined by

$$
\zeta_{2}\left(s_{1}, s_{2}\right)=\sum_{m=1}^{\infty} \frac{1}{m^{s_{1}}} \sum_{n=1}^{\infty} \frac{1}{(m+n)^{s_{2}}} .
$$

This series is absolutely convergent for $\sigma_{2}>1$ and $\sigma_{1}+\sigma_{2}>2$ (see [8, p. 419]). We can continue $\zeta_{2}\left(s_{1}, s_{2}\right)$ meromorphically to $\mathbb{C}^{2}$, which is holomorphic in

$$
\left\{\left(s_{1}, s_{2}\right) \in \mathbb{C}^{2} \mid s_{2} \neq 1, s_{1}+s_{2} \notin\{2,1,0,-2,-4,-6, \ldots\}\right\},
$$

as was proved in [1]. (The first study of the analytic continuation of $\zeta_{2}\left(s_{1}, s_{2}\right)$ is Atkinson's work [2]. Akiyama, Egami, and Tanigawa in [1] studied the analytic continuation of not only $\zeta_{2}\left(s_{1}, s_{2}\right)$ but also more general multiple zeta-functions. Zhao in [15] also obtained the continuation independently.) The special values of $\zeta_{2}\left(s_{1}, s_{2}\right)$ have been studied by various authors (see, e.g., [4], [14]).

The analytic properties of $\zeta_{2}\left(s_{1}, s_{2}\right)$ were studied by various authors (e.g., [7]-[9]). Recently, Matsumoto and Tsumura [10] studied the mean values

$$
\int_{2}^{T}\left|\zeta_{2}\left(s_{1}, s_{2}\right)\right|^{2} d t_{2}
$$

Received March 26, 2013. Revised March 28, 2014. Accepted April 4, 2014.

2010 Mathematics Subject Classification. Primary 11M32; Secondary 11M06. 
where $s_{1}$ is a fixed complex number. Theirs was the first study of the mean values of $\zeta_{2}\left(s_{1}, s_{2}\right)$. In our article we study (1.2) in the regions which are not covered in the work of Matsumoto and Tsumura and we introduce new types of mean values of $\zeta_{2}\left(s_{1}, s_{2}\right)$.

In the following we prove the next three theorems.

Theorem 1.1. Let $s_{1}=\sigma_{1}+i t_{1}$, let $s_{2}=\sigma_{2}+i t_{2} \in \mathbb{C}$, let $T \geq 2$, and let

$$
I^{[1]}(T)=\int_{2}^{T}\left|\zeta_{2}\left(s_{1}, s_{2}\right)\right|^{2} d t_{1}
$$

Assume that when $t_{1}$ moves from 2 to $T$, the point $\left(s_{1}, s_{2}\right) \in \mathbb{C}^{2}$ does not encounter the singularities of $\zeta_{2}\left(s_{1}, s_{2}\right)$. In the case $\sigma_{1}+\sigma_{2}>2$, we have

$$
I^{[1]}(T)=\zeta_{2}^{[1]}\left(2 \sigma_{1}, s_{2}\right) T+O(1)
$$

where, here and below, the implied constants depend on $\sigma_{1}, \sigma_{2}$, and $t_{2}$, and $\zeta_{2}^{[1]}\left(2 \sigma_{1}, s_{2}\right)$ is a series which converges on $\sigma_{1}+\sigma_{2}>3 / 2$. (We define $\zeta_{2}^{[1]}\left(\sigma_{1}, s_{2}\right)$ in the next section.) In the case $3 / 2<\sigma_{1}+\sigma_{2} \leq 2$, we have

$$
I^{[1]}(T)=\zeta_{2}^{[1]}\left(2 \sigma_{1}, s_{2}\right) T+ \begin{cases}O\left(T^{4-2 \sigma_{1}-2 \sigma_{2}}\right) & \left(3 / 2<\sigma_{1}+\sigma_{2}<2\right) \\ O\left((\log T)^{2}\right) & \left(\sigma_{1}+\sigma_{2}=2\right) .\end{cases}
$$

In the case $\sigma_{1}+\sigma_{2}=3 / 2$, we have

$$
I^{[1]}(T)=\left|s_{2}-1\right|^{-2} T \log T+O(T) .
$$

TheOREM 1.2. Let $s_{1}=\sigma_{1}+i t_{1}$, let $s_{2}=\sigma_{2}+i t_{2} \in \mathbb{C}$, let $T \geq 2$, and let

$$
I^{[2]}(T)=\int_{2}^{T}\left|\zeta_{2}\left(s_{1}, s_{2}\right)\right|^{2} d t_{2}
$$

Assume that when $t_{2}$ moves from 2 to $T$, the point $\left(s_{1}, s_{2}\right) \in \mathbb{C}^{2}$ does not encounter the singularities of $\zeta_{2}\left(s_{1}, s_{2}\right)$. In the case $\sigma_{2}>1$ and $\sigma_{1}+\sigma_{2}>2$, we have

$$
I^{[2]}(T)=\zeta_{2}^{[2]}\left(s_{1}, 2 \sigma_{2}\right) T+O(1),
$$

where, here and below, the implied constants depend on $\sigma_{1}, \sigma_{2}$, and $t_{1}$, and $\zeta_{2}^{[2]}\left(s_{1}, 2 \sigma_{2}\right)$ is a series which converges on $\sigma_{1}+\sigma_{2}>3 / 2$ and $\sigma_{2}>1 / 2$. 
$\left(\zeta_{2}^{[2]}\left(s_{1}, \sigma_{2}\right)\right.$ is used in [10], and we show the definition of $\zeta_{2}^{[2]}\left(s_{1}, \sigma_{2}\right)$ in the next section.) In the case $\sigma_{1}>1$ and $1 / 2<\sigma_{2} \leq 1$, we have

$$
I^{[2]}(T)=\zeta_{2}^{[2]}\left(s_{1}, 2 \sigma_{2}\right) T+ \begin{cases}O\left(T^{2-2 \sigma_{2}}\right) & \left(\sigma_{2} \neq 1\right), \\ O\left((\log T)^{2}\right) & \left(\sigma_{2}=1\right) .\end{cases}
$$

In the case $\sigma_{1} \leq 1,3 / 2<\sigma_{1}+\sigma_{2} \leq 2$, and $s_{1} \neq 1$, we have

$$
I^{[2]}(T)=\zeta_{2}^{[2]}\left(s_{1}, 2 \sigma_{2}\right) T+ \begin{cases}O\left(T^{4-2 \sigma_{1}-2 \sigma_{2}}\right) & \left(\sigma_{1}+\sigma_{2} \neq 2\right), \\ O\left((\log T)^{2}\right) & \left(\sigma_{1}+\sigma_{2}=2\right) .\end{cases}
$$

In the case $s_{1}=1$ and $1 / 2<\sigma_{2} \leq 1$, we have

$$
I^{[2]}(T)=\zeta_{2}^{[2]}\left(s_{1}, 2 \sigma_{2}\right) T+ \begin{cases}O\left(T^{2-2 \sigma_{2}}(\log T)^{2}\right) & \left(\sigma_{2} \neq 1\right) \\ O\left((\log T)^{4}\right) & \left(\sigma_{2}=1\right)\end{cases}
$$

In the case $\sigma_{1}>1$ and $\sigma_{2}=1 / 2$, we have

$$
I^{[2]}(T)=\left|\zeta\left(s_{1}\right)\right|^{2} T \log T+O(T)
$$

In the case $\sigma_{1}+\sigma_{2}=3 / 2$ and $\sigma_{2}>1 / 2$, we have

$$
I^{[2]}(T)=\left|s_{1}-1\right|^{-2} T \log T+O(T) .
$$

In the case $\sigma_{2}=1 / 2, \sigma_{1}=1$, and $s_{1} \neq 1$, we have

$$
I^{[2]}(T)=\left(\left|s_{1}-1\right|^{-2}+\left|\zeta\left(s_{1}\right)\right|^{2}\right) T \log T+O(T) .
$$

In the case $\sigma_{2}=1 / 2$ and $s_{1}=1$, we have

$$
I^{[2]}(T)=\frac{T(\log T)^{3}}{3}+O\left(T(\log T)^{2}\right) .
$$

Theorem 1.3. Let $s_{1}=\sigma_{1}+i t$, let $s_{2}=\sigma_{2}+i t \in \mathbb{C}$, let $T \geq 2$, and let

$$
I^{\square}(T)=\int_{2}^{T}\left|\zeta_{2}\left(s_{1}, s_{2}\right)\right|^{2} d t .
$$

In the case $\sigma_{2}>1$ and $\sigma_{1}+\sigma_{2}>2$, we have

$$
I^{\square}(T)=\zeta_{2}^{\square}\left(\sigma_{1}, \sigma_{2}\right) T+O(1),
$$


where, here and below, the implied constants depend on $\sigma_{1}$ and $\sigma_{2}$, and $\zeta_{2}^{\square}\left(\sigma_{1}, \sigma_{2}\right)$ is a series which converges if and only if $\sigma_{2}>1 / 2$ and $\sigma_{1}+\sigma_{2}>1$. (We define $\zeta_{2}^{\square}\left(\sigma_{1}, \sigma_{2}\right)$ in the next section.) In the case $\sigma_{1}>1$ and $1 / 2<\sigma_{2} \leq$ 1, we have

$$
I^{\square}(T)=\zeta_{2}^{\square}\left(\sigma_{1}, \sigma_{2}\right) T+O\left(T^{2-2 \sigma_{2}+\epsilon}\right)+O\left(T^{1 / 2}\right)
$$

for sufficiently small $\epsilon>0$. In the case $\sigma_{1} \leq 1$ and $3 / 2<\sigma_{1}+\sigma_{2} \leq 2$, we have

$$
I^{\square}(T)=\zeta_{2}^{\square}\left(\sigma_{1}, \sigma_{2}\right) T+O\left(T^{4-2 \sigma_{1}-2 \sigma_{2}+\epsilon}\right)+O\left(T^{1 / 2}\right)
$$

for sufficiently small $\epsilon>0$. In the case $\sigma_{1}>1$ and $\sigma_{2}=1 / 2$, we have

$$
I^{\square}(T) \sim \frac{\zeta\left(2 \sigma_{1}\right) \zeta\left(\sigma_{1}+1 / 2\right)^{2}}{\zeta\left(2 \sigma_{1}+1\right)} T \log T .
$$

Note that we can obtain $I^{[2]}(T) \sim\left|\zeta\left(s_{1}\right)\right|^{2} T \log T\left(\sigma_{1}>1, \sigma_{2}=1 / 2\right)$ by

$$
\zeta_{2}\left(s_{1}, s_{2}\right)+\zeta_{2}\left(s_{2}, s_{1}\right)=\zeta\left(s_{1}\right) \zeta\left(s_{2}\right)-\zeta\left(s_{1}+s_{2}\right)
$$

and Theorem 1.1.

Matsumoto and Tsumura [10] introduced $I^{[2]}(T)$ and studied the cases

(1) $\sigma_{1}>1$ and $\sigma_{2}>1$ in [10, Theorem 1.1],

(2) $\sigma_{1}+\sigma_{2}>2$ and $1 / 2<\sigma_{2} \leq 1$ in [10, Theorem 1.2],

(3) $1 / 2<\sigma_{1}<3 / 2,1 / 2<\sigma_{2} \leq 1$ and $3 / 2<\sigma_{1}+\sigma_{2} \leq 2$ in [10, Theorem 1.3].

They conjectured that when $\sigma_{1}+\sigma_{2}=3 / 2$, the form of the main term of the mean square formula would not be $C T$ (with a constant $C$; most probably, some log-factor would appear) (see [10, conjecture (ii), p. 385]). Our results include the regions which Matsumoto and Tsumura did not study and give an improvement on the error estimate. Moreover, by Theorem 1.2 we see that their conjecture (ii) is true.

Outlines of the proof of our theorems are as follows. We can obtain Theorems 1.1 and 1.2 by using the mean value theorems for Dirichlet polynomials and suitable approximate formulas in each theorem (see [10, Theorems 3.1 and 6.3]). The approximate formulas used in the proofs of Theorems 1.1 and 1.2 are derived from the Euler-Maclaurin formula and the simplest approximate formula to $\zeta(s)$ due to Hardy and Littlewood (see [13, p. 77]). On the other hand, we need a more elaborate method to get the proof of Theorem 1.3. To obtain the suitable approximate formula for $\zeta_{2}\left(\sigma_{1}+i t, \sigma_{2}+i t\right)$ 
we need the technique of Kiuchi and Tanigawa [6], which enables us to get good estimates of the error terms in the Euler-Maclaurin formula.

In Theorem 1.1 (resp., Theorem 1.2) we regard $s_{2}$ (resp., $s_{1}$ ) as a constant term. On the other hand, from the study of Kiuchi, Tanigawa, and Zhai [7], we know that the behavior of $\left|\zeta_{2}\left(s_{1}, s_{2}\right)\right|$ depends on both $s_{1}$ and $s_{2}$ strongly. Therefore, it is also important to consider a mean value which depends on both $s_{1}$ and $s_{2}$.

From Theorems 1.1 and 1.2 we may expect that the behavior of $\zeta_{2}\left(s_{1}, s_{2}\right)$ in the region $\sigma_{1}+\sigma_{2}=3 / 2$ is special. (Matsumoto and Tsumura in [10, Remark 1.6] conjectured that $\sigma_{1}+\sigma_{2}=3 / 2$ might be the double analogue of the critical line of the Riemann zeta-function. The error terms in Theorem 1.3 support their conjecture.) However, we can take a different point of view. For the Riemann zeta-function $\zeta(\sigma+i t)$, we know that

$$
\int_{2}^{T}|\zeta(\sigma+i t)|^{2} d t \sim \zeta(2 \sigma) T
$$

$($ for $\sigma>1 / 2)$ and

$$
\int_{2}^{T}|\zeta(1 / 2+i t)|^{2} d t \sim T \log T
$$

hold (see, e.g., [13, Theorems 7.2 and 7.3]). The line $\sigma=1 / 2$ is the critical line for $\zeta(\sigma+i t)$, and the series

$$
\zeta(2 \sigma)=\sum_{n=1}^{\infty} \frac{1}{n^{2 \sigma}}
$$

diverges on $\sigma=1 / 2$. On the other hand, $\zeta_{2}^{\square}\left(\sigma_{1}, \sigma_{2}\right)$ converges if and only if $\sigma_{2}>1 / 2$ and $\sigma_{1}+\sigma_{2}>1$. Moreover, if $\sigma_{1}=\sigma_{2}>1 / 2$, then $I^{\square}(T) \sim$ $\zeta_{2}^{\square}\left(\sigma_{1}, \sigma_{2}\right) T$ holds by

$$
\int_{2}^{T}|\zeta(\sigma+i t)|^{4} d t=O(T)
$$

for $\sigma>1 / 2$ (see [13, Theorem 7.5]) and Carlson's mean value theorem (see $[12$, p. 304] $)$. Hence, we can expect that $I^{\square}(T) \sim \zeta_{2}^{\square}\left(\sigma_{1}, \sigma_{2}\right) T$ holds for $\sigma_{2}>$ $1 / 2$ and $\sigma_{1}+\sigma_{2}>1$ and that the boundary of the region $\sigma_{2}>1 / 2$ and $\sigma_{1}+\sigma_{2}>1$ is an analogue of the critical line for $\zeta_{2}\left(\sigma_{1}+i t, \sigma_{2}+i t\right)$. 


\section{§2. Lemmas for the proof of theorems}

In this section, we collect some auxiliary results and definitions.

First, we give the definition of $\zeta_{2}^{[1]}\left(\sigma_{1}, s_{2}\right), \zeta_{2}^{[2]}\left(s_{1}, \sigma_{2}\right)$, and $\zeta_{2}^{\square}\left(\sigma_{1}, \sigma_{2}\right)$.

We define

$$
\zeta_{2}^{[1]}\left(\sigma_{1}, s_{2}\right)=\sum_{m=1}^{\infty} \frac{1}{m^{\sigma_{1}}}\left|\zeta\left(s_{2}\right)-\sum_{n=1}^{m} \frac{1}{n^{s_{2}}}\right|^{2}
$$

for $s_{2} \neq 1$. Since we have

$$
\zeta_{2}^{[1]}\left(2 \sigma_{1}, s_{2}\right) \ll \sum_{m=1}^{\infty} \begin{cases}m^{2-2 \sigma_{1}-2 \sigma_{2}} & \left(\sigma_{2}>1\right), \\ m^{-2 \sigma_{1}}(\log m)^{2} & \left(\sigma_{2}=1\right), \\ m^{2-2 \sigma_{1}-2 \sigma_{2}} & \left(\sigma_{2}<1\right),\end{cases}
$$

the series $\zeta_{2}^{[1]}\left(2 \sigma_{1}, s_{2}\right)$ converges in the region $\sigma_{1}+\sigma_{2}>3 / 2$.

We define

$$
\zeta_{2}^{[2]}\left(s_{1}, \sigma_{2}\right)=\sum_{n=2}^{\infty}\left|\sum_{m=1}^{n-1} \frac{1}{m^{s_{1}}}\right|^{2} \frac{1}{n^{\sigma_{2}}} .
$$

(This definition is the same as $[10,(1.2)]$.$) Since we have$

$$
\zeta_{2}^{[2]}\left(s_{1}, 2 \sigma_{2}\right) \ll \sum_{n=2}^{\infty} \begin{cases}n^{-2 \sigma_{2}} & \left(\sigma_{1}>1\right), \\ n^{-2 \sigma_{2}}(\log n)^{2} & \left(\sigma_{1}=1\right), \\ n^{2-2 \sigma_{1}-2 \sigma_{2}} & \left(\sigma_{1}<1\right),\end{cases}
$$

the series $\zeta_{2}^{[2]}\left(s_{1}, 2 \sigma_{2}\right)$ converges in the region $\sigma_{2}>1 / 2$ and $\sigma_{1}+\sigma_{2}>3 / 2$.

We define

$$
\zeta_{2}^{\square}\left(\sigma_{1}, \sigma_{2}\right)=\sum_{k=2}^{\infty}\left(\sum_{\substack{m n=k \\ m<n}} \frac{1}{m^{\sigma_{1}} n^{\sigma_{2}}}\right)^{2} .
$$

We note that $\#\{(m, n) \mid m n=k, m<n\} \ll k^{\epsilon}$ for any $\epsilon>0$. Since

$$
\begin{aligned}
\zeta_{2}\left(2 \sigma_{1}, 2 \sigma_{2}\right) & <\zeta_{2}^{\square}\left(\sigma_{1}, \sigma_{2}\right) \\
& =\sum_{k=2}^{\infty} k^{-2 \sigma_{2}}\left(\sum_{\substack{m \mid k \\
m<\sqrt{k}}} \frac{1}{m^{\sigma_{1}-\sigma_{2}}}\right)^{2} \\
& \ll \sum_{k=2}^{\infty} \begin{cases}k^{-2 \sigma_{2}+\epsilon} & \left(\sigma_{1} \geq \sigma_{2}\right), \\
k^{-\sigma_{1}-\sigma_{2}+\epsilon} & \left(\sigma_{1}<\sigma_{2}\right)\end{cases}
\end{aligned}
$$


for any $\epsilon>0$, the series $\zeta_{2}^{\square}\left(\sigma_{1}, \sigma_{2}\right)$ converges if and only if $\sigma_{2}>1 / 2$ and $\sigma_{1}+\sigma_{2}>1$.

Lemma 2.1 ([5, Theorem 5.2]). Let $a_{1}, \ldots, a_{N}$ be arbitrary complex numbers. Then

$$
\int_{0}^{T}\left|\sum_{n \leq N} a_{n} n^{i t}\right|^{2} d t=T \sum_{n \leq N}\left|a_{n}\right|^{2}+O\left(\sum_{n \leq N} n\left|a_{n}\right|^{2}\right),
$$

which also remains valid if $N=\infty$, provided that the series on the righthand side of (2.4) converges.

The following lemmas are well-known results for $\zeta(s)$ (see [3, p. 114] and [13, Theorem 4.11]).

Lemma 2.2. Let $s=\sigma+i t \in \mathbb{C}$, let $m \in \mathbb{N} \cup\{0\}$, let $N \in \mathbb{N}$, and let $M=$ $2 m+1$. For $\sigma>-2 m$ we have

$$
\begin{aligned}
\zeta(s)= & \sum_{n \leq N} \frac{1}{n^{s}}+\frac{N^{1-s}}{s-1}-\frac{N^{-s}}{2} \\
& +\sum_{k=1}^{2 m} \frac{B_{k+1}}{(k+1) !}(s)_{k} N^{-(s+k)}+R_{M, N}(s),
\end{aligned}
$$

where $(s)_{k}=s(s+1) \cdots(s+k-1)$ and

$$
R_{M, N}(s)=-\frac{(s)_{M}}{M !} \int_{N}^{\infty} B_{M}(x-[x]) x^{-s-M} d x .
$$

Corollary 2.1. Let $s=1+i t$. For fixed $t>0$ we have

$$
\zeta(s)-\sum_{n \leq N} \frac{1}{n^{s}}=\frac{N^{1-s}}{s-1}+O\left(N^{-1}\right)=O(1)
$$

where the implied constants do not depend on $N$.

Lemma 2.3. Let $s=\sigma+i t \in \mathbb{C}$. We have

$$
\zeta(s)=\sum_{1 \leq n \leq x} \frac{1}{n^{s}}-\frac{x^{1-s}}{1-s}+O\left(x^{-\sigma}\right)
$$

uniformly for $\sigma \geq \sigma_{0}>0, x \geq 1$, and $|t| \leq 2 \pi x / C$, where $C$ is a given constant greater than 1 . 
We use the following evaluations in this paper.

REMARK 2.1. Let $T \geq 1$, and let $M \geq 1$, with $M \ll \log T$. For fixed $\alpha, \beta \geq$ 0 we have

$$
\begin{aligned}
\sum_{k \leq M}\left(\frac{T}{2^{k}}\right)^{\alpha}\left(\log \left(\frac{T}{2^{k}}\right)\right)^{\beta} & \ll T^{\alpha} \sum_{k \leq M}\left(\frac{1}{2^{\alpha}}\right)^{k}\left((\log T)^{\beta}+k^{\beta}\right) \\
& \ll \begin{cases}T^{\alpha}(\log T)^{\beta} & (\alpha \neq 0), \\
(\log T)^{\beta+1} & (\alpha=0) .\end{cases}
\end{aligned}
$$

\section{§3. Proof of Theorem 1.1}

In this section, we regard $\sigma_{1}, s_{2}$ as constants. We divide the proof into two cases.

Proof of Theorem 1.1 for $\sigma_{1}+\sigma_{2}>2$. We set

$$
a_{m}=\frac{1}{m^{\sigma_{1}}}\left(\zeta\left(s_{2}\right)-\sum_{n=1}^{m} \frac{1}{n^{s_{2}}}\right)
$$

for $m \in \mathbb{N}$. If we assume that $\sigma_{2}>1$, then we have

$$
\begin{aligned}
\zeta_{2}\left(s_{1}, s_{2}\right) & =\sum_{m=1}^{\infty} \frac{1}{m^{\sigma_{1}+i t_{1}}} \sum_{n=m+1}^{\infty} \frac{1}{n^{s_{2}}} \\
& =\sum_{m=1}^{\infty} a_{m} m^{-i t_{1}} .
\end{aligned}
$$

The last series converges absolutely in $\sigma_{1}+\sigma_{2}>2$. Since

$$
\sum_{m=1}^{\infty} m\left|a_{m}\right|^{2}=\sum_{m=1}^{\infty} \frac{1}{m^{2 \sigma_{1}-1}}\left|\zeta\left(s_{2}\right)-\sum_{n=1}^{m} \frac{1}{n^{s_{2}}}\right|^{2}
$$

converges by (2.1), we have

$$
I^{[1]}(T)=\zeta_{2}^{[1]}\left(2 \sigma_{1}, s_{2}\right) T+O(1)
$$

by Lemma 2.1 .

In the case $3 / 2 \leq \sigma_{1}+\sigma_{2} \leq 2$, we use the following lemma. 
LEMMA 3.1. Let $s_{1}=\sigma_{1}+i t_{1}$ with $t_{1} \geq 1$, let $s_{2}=\sigma_{2}+i t_{2} \in \mathbb{C}$, and let $N \in \mathbb{N}$. Let $C>1$ be a given constant. Assume that the point $\left(s_{1}, s_{2}\right) \in \mathbb{C}^{2}$ does not encounter the singularities of $\zeta_{2}\left(s_{1}, s_{2}\right)$. If $1<\left|t_{1}+t_{2}\right|<2 \pi N / C$, then we have

$$
\zeta_{2}\left(s_{1}, s_{2}\right)=\sum_{m \leq N} \frac{1}{m^{s_{1}}}\left(\zeta\left(s_{2}\right)-\sum_{n=1}^{m} \frac{1}{n^{s_{2}}}\right)+O\left(t_{1}^{-1} N^{2-\sigma_{1}-\sigma_{2}}\right)
$$

for $\sigma_{1}+\sigma_{2}>1$ and any fixed $\sigma_{1}, s_{2}$.

Proof. Let $l \in \mathbb{N}$ with $\sigma_{2}>-2 l$. To obtain the analytic continuation of $\zeta_{2}\left(s_{1}, s_{2}\right)$, we regard $s_{1}$ and $s_{2}$ as complex variables, and we assume that $\sigma_{1}, \sigma_{2}>1$ temporarily. For any $N \in \mathbb{N}$, we have, say,

$$
\zeta_{2}\left(s_{1}, s_{2}\right)=\sum_{m=1}^{N} \frac{1}{m^{s_{1}}} \sum_{n=m+1}^{\infty} \frac{1}{n^{s_{2}}}+\sum_{m=N+1}^{\infty} \frac{1}{m^{s_{1}}} \sum_{n=m+1}^{\infty} \frac{1}{n^{s_{2}}}=V_{1}+V_{2} .
$$

Since

$$
V_{1}=\sum_{m=1}^{N} \frac{1}{m^{s_{1}}}\left(\zeta\left(s_{2}\right)-\sum_{n=1}^{m} \frac{1}{n^{s_{2}}}\right)
$$

$V_{1}$ is continued meromorphically to $\mathbb{C}^{2}$. By setting $M=2 l+1$ in Lemma 2.2, we have, say,

$$
\begin{aligned}
V_{2}= & \sum_{m=N+1}^{\infty} \frac{1}{m^{s_{1}}}\left(\frac{m^{1-s_{2}}}{s_{2}-1}-\frac{m^{-s_{2}}}{2}+\sum_{k=1}^{M-1} \frac{B_{k+1}}{(k+1) !}\left(s_{2}\right)_{k} m^{-s_{2}-k}+R_{M, m}\left(s_{2}\right)\right) \\
= & \frac{1}{s_{2}-1} \sum_{m=N+1}^{\infty} \frac{1}{m^{s_{1}+s_{2}-1}}-\frac{1}{2} \sum_{m=N+1}^{\infty} \frac{1}{m^{s_{1}+s_{2}}} \\
& +\sum_{k=1}^{M-1} \frac{B_{k+1}}{(k+1) !}\left(s_{2}\right)_{k} \sum_{m=N+1}^{\infty} \frac{1}{m^{s_{1}+s_{2}+k}}+\sum_{m=N+1}^{\infty} \frac{1}{m^{s_{1}}} R_{M, m}\left(s_{2}\right) \\
= & \frac{1}{s_{2}-1}\left(\zeta\left(s_{1}+s_{2}-1\right)-\sum_{m=1}^{N} \frac{1}{m^{s_{1}+s_{2}-1}}\right)-\frac{1}{2} \sum_{m=N+1}^{\infty} \frac{1}{m^{s_{1}+s_{2}}} \\
& +\sum_{k=1}^{M-1} \frac{B_{k+1}}{(k+1) !}\left(s_{2}\right)_{k} \sum_{m=N+1}^{\infty} \frac{1}{m^{s_{1}+s_{2}+k}}+\sum_{m=N+1}^{\infty} \frac{1}{m^{s_{1}}} R_{M, m}\left(s_{2}\right) \\
= & I_{1}+I_{2}+I_{3}+I_{4} .
\end{aligned}
$$


Since $I_{4}$ converges absolutely for $\sigma_{2}>-M+1=-2 l$ and $\sigma_{1}+\sigma_{2}>-1, V_{2}$ is continued meromorphically to $\sigma_{2}>-2 l$ and $\sigma_{1}+\sigma_{2}>1$. Now, we regard $\sigma_{1}, s_{2}$ as constants. By Lemma 2.3, we have $I_{1} \ll t_{1}^{-1} N^{2-\sigma_{1}-\sigma_{2}}$. Also, we can easily obtain $I_{2}, I_{3}, I_{4} \ll t_{1}^{-1} N^{2-\sigma_{1}-\sigma_{2}}$. This implies the lemma.

Proof of Theorem 1.1 for $3 / 2 \leq \sigma_{1}+\sigma_{2} \leq 2$. Let

$$
a_{m}=m^{-\sigma_{1}}\left(\zeta\left(s_{2}\right)-\sum_{n=1}^{m} n^{-s_{2}}\right)
$$

and let

$$
m_{0}=\max \left\{m \in \mathbb{N}\left|\frac{T}{2^{m}}>\right| t_{2} \mid+1\right\}
$$

Note that

$$
\sum_{m=1}^{\infty}\left|a_{m}\right|^{2}=\zeta_{2}^{[1]}\left(2 \sigma_{1}, s_{2}\right)
$$

(in the case $\sigma_{1}+\sigma_{2}>3 / 2$ ) and that

$$
m_{0}<\frac{\log T-\log \left(\left|t_{2}\right|+1\right)}{\log 2} \leq m_{0}+1
$$

hold. We take $T \geq 2$ and $N \in \mathbb{N}$ with $\left|t_{2}\right|+1<T$ and $3 T<2 \pi N / C$, where $C>1$, and we assume that $T<t_{1}<2 T$. Then we have

$$
1<t_{1}-\left|t_{2}\right|<\left|t_{1}+t_{2}\right|<\left|t_{1}\right|+\left|t_{2}\right|<3 T<\frac{2 \pi N}{C} .
$$

Therefore, we can use Lemma 3.1, and we have, say,

$$
\zeta_{2}\left(s_{1}, s_{2}\right)=\sum_{m=1}^{N} a_{m} m^{-i t_{1}}+O\left(t_{1}^{-1} N^{2-\sigma_{1}-\sigma_{2}}\right)=I_{1}+I_{2} .
$$

Since $a_{m} \ll m^{-\sigma_{1}-\sigma_{2}+1}$ by Corollary 2.1 , we obtain

$$
\sum_{m=1}^{N} m a_{m}^{2} \ll \sum_{m=1}^{N} m^{3-2 \sigma_{1}-2 \sigma_{2}} \ll \begin{cases}\log N & \left(\sigma_{1}+\sigma_{2}=2\right), \\ N^{4-2 \sigma_{1}-2 \sigma_{2}} & \left(\sigma_{1}+\sigma_{2}<2\right)\end{cases}
$$

and

$$
I_{1} \ll \sum_{m=1}^{N} a_{m} \ll \sum_{m=1}^{N} m^{1-\sigma_{1}-\sigma_{2}} \ll \begin{cases}\log N & \left(\sigma_{1}+\sigma_{2}=2\right), \\ N^{2-\sigma_{1}-\sigma_{2}} & \left(\sigma_{1}+\sigma_{2}<2\right) .\end{cases}
$$


Therefore, we have

$$
\int_{T}^{2 T}\left|I_{1}\right|^{2} d t_{1}=T \sum_{m=1}^{N}\left|a_{m}\right|^{2}+ \begin{cases}O(\log N) & \left(\sigma_{1}+\sigma_{2}=2\right) \\ O\left(N^{4-2 \sigma_{1}-2 \sigma_{2}}\right) & \left(\sigma_{1}+\sigma_{2}<2\right)\end{cases}
$$

by Lemma 2.1 and

$$
\begin{aligned}
\int_{T}^{2 T}\left|I_{1} I_{2}\right| d t_{1} & \ll N^{2-\sigma_{1}-\sigma_{2}} \max _{T<t_{1}<2 T}\left|I_{1}\right| \\
& \ll \begin{cases}\log N & \left(\sigma_{1}+\sigma_{2}=2\right), \\
N^{4-2 \sigma_{1}-2 \sigma_{2}} & \left(\sigma_{1}+\sigma_{2}<2\right) .\end{cases}
\end{aligned}
$$

On the other hand, we have

$$
\int_{T}^{2 T}\left|I_{2}\right|^{2} d t_{1} \ll N^{4-2 \sigma_{1}-2 \sigma_{2}} \int_{T}^{2 T} \frac{d t_{1}}{t_{1}^{2}} \ll T^{-1} N^{4-2 \sigma_{1}-2 \sigma_{2}} .
$$

Therefore, we have

$$
\begin{aligned}
& \int_{T}^{2 T}\left|\zeta_{2}\left(s_{1}, s_{2}\right)\right|^{2} d t_{1} \\
& \quad=T \sum_{m=1}^{N}\left|a_{m}\right|^{2}+ \begin{cases}O(\log N) & \left(\sigma_{1}+\sigma_{2}=2\right) \\
O\left(N^{4-2 \sigma_{1}-2 \sigma_{2}}\right) & \left(\sigma_{1}+\sigma_{2}<2\right)\end{cases}
\end{aligned}
$$

By setting $N=[T]+1$, we obtain

$$
\begin{aligned}
& \int_{T}^{2 T}\left|\zeta_{2}\left(s_{1}, s_{2}\right)\right|^{2} d t_{1} \\
& \quad=T \sum_{m \leq T}\left|a_{m}\right|^{2}+ \begin{cases}O(\log T) & \left(\sigma_{1}+\sigma_{2}=2\right) \\
O\left(T^{4-2 \sigma_{1}-2 \sigma_{2}}\right) & \left(\sigma_{1}+\sigma_{2}<2\right)\end{cases}
\end{aligned}
$$

Therefore, in the case $\sigma_{1}+\sigma_{2}>3 / 2$, we have

$$
\begin{aligned}
& \int_{T}^{2 T}\left|\zeta_{2}\left(s_{1}, s_{2}\right)\right|^{2} d t_{1} \\
& \quad=\zeta_{2}^{[1]}\left(2 \sigma_{1}, s_{2}\right) T+ \begin{cases}O(\log T) & \left(\sigma_{1}+\sigma_{2}=2\right), \\
O\left(T^{4-2 \sigma_{1}-2 \sigma_{2}}\right) & \left(3 / 2<\sigma_{1}+\sigma_{2}<2\right) .\end{cases}
\end{aligned}
$$


By this relation and Remark 2.1, we obtain

$$
\begin{aligned}
& \int_{\left|t_{2}\right|+1}^{T}\left|\zeta_{2}\left(s_{1}, s_{2}\right)\right|^{2} d t_{1} \\
& =\int_{T / 2^{m_{0}}}^{T}\left|\zeta_{2}\left(s_{1}, s_{2}\right)\right|^{2} d t_{1}+O(1) \\
& =\sum_{1 \leq k \leq m_{0}} \int_{T / 2^{k}}^{T / 2^{k-1}}\left|\zeta_{2}\left(s_{1}, s_{2}\right)\right|^{2} d t_{1}+O(1) \\
& =\zeta_{2}^{[1]}\left(2 \sigma_{1}, s_{2}\right) T \sum_{1 \leq k \leq m_{0}} \frac{1}{2^{k}} \\
& + \begin{cases}O\left(\sum_{1 \leq k \leq m_{0}} \log \frac{T}{2^{k}}\right) & \left(\sigma_{1}+\sigma_{2}=2\right), \\
O\left(\sum_{1 \leq k \leq m_{0}}\left(\frac{T}{2^{k}}\right)^{4-2 \sigma_{1}-2 \sigma_{2}}\right) & \left(3 / 2<\sigma_{1}+\sigma_{2}<2\right)\end{cases} \\
& =\zeta_{2}^{[1]}\left(2 \sigma_{1}, s_{2}\right) T+ \begin{cases}O\left((\log T)^{2}\right) & \left(\sigma_{1}+\sigma_{2}=2\right), \\
O\left(T^{4-2 \sigma_{1}-2 \sigma_{2}}\right) & \left(3 / 2<\sigma_{1}+\sigma_{2}<2\right) .\end{cases}
\end{aligned}
$$

This implies the theorem for $3 / 2<\sigma_{1}+\sigma_{2} \leq 2$.

In the case $\sigma_{1}+\sigma_{2}=3 / 2$, since

$$
a_{m}=m^{-\sigma_{1}}\left(\zeta\left(s_{2}\right)-\sum_{n=1}^{m} n^{-s_{2}}\right)=\frac{m^{1-\sigma_{1}-s_{2}}}{s_{2}-1}+O\left(m^{-\sigma_{1}-\sigma_{2}}\right)
$$

by Lemma 2.2, we have

$$
\left|a_{m}\right|^{2}=\frac{m^{-1}}{\left|s_{2}-1\right|^{2}}+O\left(m^{-2}\right)
$$

Therefore, we have

$$
\int_{T}^{2 T}\left|\zeta_{2}\left(s_{1}, s_{2}\right)\right|^{2} d t_{1}=\frac{T \log T}{\left|s_{2}-1\right|^{2}}+O(T)
$$

by (3.1). By Remark 2.1 and this relation, we obtain the theorem.

\section{§4. Proof of Theorem 1.2}

In this section, we regard $\sigma_{2}, s_{1}$ as constants. We divide the proof into three cases. 
Proof of Theorem 1.2 for $\sigma_{2}>1$ and $\sigma_{1}+\sigma_{2}>2$. We set

$$
a_{n}=\frac{1}{n^{\sigma_{2}}} \sum_{m=1}^{n-1} \frac{1}{m^{s_{1}}}
$$

for $n \in \mathbb{N}$. We have

$$
\zeta_{2}\left(s_{1}, s_{2}\right)=\sum_{n=2}^{\infty}\left(\sum_{m=1}^{n-1} \frac{1}{m^{s_{1}}}\right) \frac{1}{n^{\sigma_{2}+i t_{2}}}=\sum_{n=2}^{\infty} a_{n} n^{-i t_{2}} .
$$

Since

$$
\sum_{n=2}^{\infty} n\left|a_{n}\right|^{2}=\sum_{n=2}^{\infty}\left(\sum_{m=1}^{n-1} \frac{1}{m^{s_{1}}}\right) \frac{1}{n^{2 \sigma_{2}-1}}
$$

converges by (2.2), we have

$$
I^{[2]}(T)=\zeta_{2}^{[2]}\left(s_{1}, 2 \sigma_{2}\right) T+O(1)
$$

by Lemma 2.1.

We use the following lemma in the cases $\sigma_{1}>1,1 / 2<\sigma_{2} \leq 1$ and $\sigma_{1} \leq 1$, $3 / 2 \leq \sigma_{1}+\sigma_{2} \leq 2$.

LEMMA 4.1. Let $s_{1}=\sigma_{1}+i t_{1}$, let $s_{2}=\sigma_{2}+i t_{2} \in \mathbb{C}$ with $t_{2} \geq 1$, and let $N \in \mathbb{N}$ with $N>e^{2}$. Let $C>1$ be a given constant. Assume that the point $\left(s_{1}, s_{2}\right) \in \mathbb{C}^{2}$ does not encounter the singularities of $\zeta_{2}\left(s_{1}, s_{2}\right)$. If $1<t_{2}<$ $2 \pi N / C$ and $1<\left|t_{1}+t_{2}\right|<2 \pi N / C$, then we have

$$
\begin{aligned}
\zeta_{2}\left(s_{1}, s_{2}\right)= & \sum_{2 \leq n \leq N}\left(\sum_{m=1}^{n-1} \frac{1}{m^{s_{1}}}\right) \frac{1}{n^{s_{2}}} \\
& + \begin{cases}O\left(t_{2}^{-1} N^{1-\sigma_{2}}+t_{2}^{-1} N^{2-\sigma_{1}-\sigma_{2}}\right) & \left(s_{1} \neq 1\right), \\
O\left(t_{2}^{-1} N^{1-\sigma_{2}} \log N\right) & \left(s_{1}=1\right)\end{cases}
\end{aligned}
$$

for $\sigma_{2} \geq 1 / 2, \sigma_{1}+\sigma_{2}>1$ and any fixed $\sigma_{2}, s_{1}$.

Proof. Let $l \in \mathbb{N}$ with $\sigma_{1}>-2 l$. To obtain the analytic continuation of $\zeta_{2}\left(s_{1}, s_{2}\right)$, we regard $s_{1}$ and $s_{2}$ as complex variables and assume that $\sigma_{1}, \sigma_{2}>$ 1 temporarily. For any $N \in \mathbb{N}$, we have, say,

$$
\zeta_{2}\left(s_{1}, s_{2}\right)=\sum_{2 \leq n \leq N}\left(\sum_{m=1}^{n-1} \frac{1}{m^{s_{1}}}\right) \frac{1}{n^{s_{2}}}+\sum_{n>N}\left(\sum_{m=1}^{n-1} \frac{1}{m^{s_{1}}}\right)=U_{1}+U_{2} .
$$


The term $U_{1}$ is obviously holomorphic in $\mathbb{C}^{2}$. By setting $M=2 l+1$ in Lemma 2.2, we have, say,

$$
\begin{aligned}
& U_{2}=\sum_{n>N}\left(\sum_{m=1}^{n} \frac{1}{m^{s_{1}}}-\frac{1}{n^{s_{1}}}\right) \frac{1}{n^{s_{2}}} \\
& =\sum_{n>N}\left(\zeta\left(s_{1}\right)-\frac{n^{1-s_{1}}}{s_{1}-1}-\frac{n^{-s_{1}}}{2}\right. \\
& \left.-\sum_{k=1}^{M-1} \frac{B_{k+1}}{(k+1) !}\left(s_{1}\right)_{k} n^{-s_{1}-k}-R_{M, n}\left(s_{1}\right)\right) \frac{1}{n^{s_{2}}} \\
& =\zeta\left(s_{1}\right) \sum_{n>N} \frac{1}{n^{s_{2}}}+\frac{1}{1-s_{1}} \sum_{n>N} \frac{1}{n^{s_{1}+s_{2}-1}}-\frac{1}{2} \sum_{n>N} \frac{1}{n^{s_{1}+s_{2}}} \\
& -\sum_{k=1}^{M-1} \frac{B_{k+1}}{(k+1) !}\left(s_{1}\right)_{k} \sum_{n>N} \frac{1}{n^{s_{1}+s_{2}+k}}-\sum_{n>N} \frac{1}{n^{s_{2}}} R_{M, n}\left(s_{1}\right) \\
& =\zeta\left(s_{1}\right)\left(\zeta\left(s_{2}\right)-\sum_{n=1}^{N} \frac{1}{n^{s_{2}}}\right) \\
& +\frac{1}{1-s_{1}}\left(\zeta\left(s_{1}+s_{2}-1\right)-\sum_{n=1}^{N} \frac{1}{n^{s_{1}+s_{2}-1}}\right) \\
& -\frac{1}{2} \sum_{n>N} \frac{1}{n^{s_{1}+s_{2}}}-\sum_{k=1}^{M-1} \frac{B_{k+1}}{(k+1) !}\left(s_{1}\right)_{k} \sum_{n>N} \frac{1}{n^{s_{1}+s_{2}+k}} \\
& -\sum_{n>N} \frac{1}{n^{s_{2}}} R_{M, n}\left(s_{1}\right) \\
& =I_{1}+I_{2}+I_{3}+I_{4}+I_{5} \text {. }
\end{aligned}
$$

Since $I_{5}$ converges absolutely for $\sigma_{1}>-M+1=-2 l$ and $\sigma_{1}+\sigma_{2}>-1, U_{2}$ is continued meromorphically to $\sigma_{2}>0, \sigma_{1}>-2 l$, and $\sigma_{1}+\sigma_{2}>1$. Now, we regard $\sigma_{2}, s_{1}$ as constants.

In the case $s_{1} \neq 1$, by Lemma 2.3 we have $I_{1} \ll\left|s_{1}-1\right|^{-1} t_{2}^{-1} N^{1-\sigma_{2}}$ and $I_{2} \ll\left|s_{1}-1\right|^{-1} t_{2}^{-1} N^{2-\sigma_{1}-\sigma_{2}}$. Also, we can easily obtain $I_{3}, I_{4}, I_{5} \ll$ $t_{2}^{-1} N^{2-\sigma_{1}-\sigma_{2}}$. This implies the lemma for $s_{1} \neq 1$. In the case $s_{1}=1$, we obtain the lemma by using the maximum modulus principle. 
We prove Theorem 1.2 for $\sigma_{1}>1,1 / 2 \leq \sigma_{2} \leq 1$ or $\sigma_{1} \leq 1,3 / 2 \leq$ $\sigma_{1}+\sigma_{2} \leq 2$. We divide the proof into the case $s_{1} \neq 1$ and the case $s_{1}=1$.

Proof of Theorem 1.2 for $s_{1} \neq 1$. We prove the theorem by the same argument as in the proof of Theorem 1.1.

Let

$$
a_{n}=n^{-\sigma_{2}} \sum_{m=1}^{n-1} m^{-s_{1}}
$$

Note that

$$
\sum_{n=2}^{\infty}\left|a_{n}\right|^{2}=\zeta_{2}^{[2]}\left(s_{1}, 2 \sigma_{2}\right)
$$

in the case $\sigma_{1}+\sigma_{2}>3 / 2$ and $\sigma_{2}>1 / 2$. We take $T \geq 2$ and $N \in \mathbb{N}$ with $N>e^{2},\left|t_{1}\right|+1<T$, and $3 T<2 \pi N / C$, where $C>1$, and we assume that $T<t_{2}<2 T$. Then we can use Lemma 4.1, and we have, say,

$$
\zeta_{2}\left(s_{1}, s_{2}\right)=\sum_{n=2}^{N} a_{n} n^{-i t_{2}}+O\left(t_{2}^{-1} N^{1-\sigma_{2}}+t_{2}^{-1} N^{2-\sigma_{1}-\sigma_{2}}\right)=I_{1}+I_{2} .
$$

Since

$$
a_{n} \ll \begin{cases}n^{-\sigma_{2}} & \left(\sigma_{1} \geq 1\right), \\ n^{-\sigma_{1}-\sigma_{2}+1} & \left(\sigma_{1}<1\right)\end{cases}
$$

by Corollary 2.1, we obtain

$$
\sum_{n=2}^{N} n a_{n}^{2} \ll \begin{cases}\log N & \left(\sigma_{2}=1, \sigma_{1} \geq 1\right), \\ N^{2-2 \sigma_{2}} & \left(\sigma_{2}<1, \sigma_{1} \geq 1\right), \\ \log N & \left(\sigma_{1}+\sigma_{2}=2, \sigma_{1}<1\right), \\ N^{4-2 \sigma_{1}-2 \sigma_{2}} & \left(\sigma_{1}+\sigma_{2}<2, \sigma_{1}<1\right)\end{cases}
$$

and

$$
I_{1} \ll \sum_{n=2}^{N} a_{n} \ll \begin{cases}\log N & \left(\sigma_{2}=1, \sigma_{1} \geq 1\right), \\ N^{1-\sigma_{2}} & \left(\sigma_{2}<1, \sigma_{1} \geq 1\right), \\ \log N & \left(\sigma_{1}+\sigma_{2}=2, \sigma_{1}<1\right), \\ N^{2-\sigma_{1}-\sigma_{2}} & \left(\sigma_{1}+\sigma_{2}<2, \sigma_{1}<1\right) .\end{cases}
$$


Therefore, we have

$$
\int_{T}^{2 T}\left|I_{1}\right|^{2} d t_{2}=T \sum_{n=2}^{N}\left|a_{n}\right|^{2}+ \begin{cases}O(\log N) & \left(\sigma_{2}=1, \sigma_{1} \geq 1\right) \\ O\left(N^{2-2 \sigma_{2}}\right) & \left(\sigma_{2}<1, \sigma_{1} \geq 1\right) \\ O(\log N) & \left(\sigma_{1}+\sigma_{2}=2, \sigma_{1}<1\right) \\ O\left(N^{4-2 \sigma_{1}-2 \sigma_{2}}\right) & \left(\sigma_{1}+\sigma_{2}<2, \sigma_{1}<1\right)\end{cases}
$$

by Lemma 2.1 and

$$
\begin{aligned}
\int_{T}^{2 T}\left|I_{1} I_{2}\right| d t_{2} \ll & \begin{cases}\left(N^{1-\sigma_{2}}+N^{2-\sigma_{1}-\sigma_{2}}\right) \log N & \left(\sigma_{2}=1, \sigma_{1} \geq 1\right), \\
\left(N^{1-\sigma_{2}}+N^{2-\sigma_{1}-\sigma_{2}}\right) N^{1-\sigma_{2}} & \left(\sigma_{2}<1, \sigma_{1} \geq 1\right), \\
\left(N^{1-\sigma_{2}}+N^{2-\sigma_{1}-\sigma_{2}}\right) \log N & \left(\sigma_{1}+\sigma_{2}=2, \sigma_{1}<1\right), \\
\left(N^{1-\sigma_{2}}+N^{2-\sigma_{1}-\sigma_{2}}\right) N^{2-\sigma_{1}-\sigma_{2}} & \left(\sigma_{1}+\sigma_{2}<2, \sigma_{1}<1\right)\end{cases} \\
& \ll \begin{cases}\log N & \left(\sigma_{2}=1, \sigma_{1} \geq 1\right), \\
N^{2-2 \sigma_{2}} & \left(\sigma_{2}<1, \sigma_{1} \geq 1\right), \\
\log N & \left(\sigma_{1}+\sigma_{2}=2, \sigma_{1}<1\right), \\
N^{4-2 \sigma_{1}-2 \sigma_{2}} & \left(\sigma_{1}+\sigma_{2}<2, \sigma_{1}<1\right) .\end{cases}
\end{aligned}
$$

On the other hand, we have

$$
\int_{T}^{2 T}\left|I_{2}\right|^{2} d t_{2} \ll T^{-1}\left(N^{2-2 \sigma_{2}}+N^{4-2 \sigma_{1}-2 \sigma_{2}}\right) .
$$

Therefore, we have

$$
\int_{T}^{2 T}\left|\zeta_{2}\left(s_{1}, s_{2}\right)\right|^{2} d t_{2}=T \sum_{n=2}^{N}\left|a_{n}\right|^{2}+ \begin{cases}O(\log N) & \left(\sigma_{2}=1, \sigma_{1} \geq 1\right), \\ O\left(N^{2-2 \sigma_{2}}\right) & \left(\sigma_{2}<1, \sigma_{1} \geq 1\right), \\ O(\log N) & \left(\sigma_{1}+\sigma_{2}=2, \sigma_{1}<1\right), \\ O\left(N^{4-2 \sigma_{1}-2 \sigma_{2}}\right) & \left(\sigma_{1}+\sigma_{2}<2, \sigma_{1}<1\right) .\end{cases}
$$

By setting $N=[T]+1$, we obtain

$$
\begin{aligned}
& \int_{T}^{2 T}\left|\zeta_{2}\left(s_{1}, s_{2}\right)\right|^{2} d t_{2} \\
& \quad=T \sum_{n \leq T}\left|a_{n}\right|^{2}+ \begin{cases}O(\log T) & \left(\sigma_{2}=1, \sigma_{1} \geq 1\right), \\
O\left(T^{2-2 \sigma_{2}}\right) & \left(\sigma_{2}<1, \sigma_{1} \geq 1\right), \\
O(\log T) & \left(\sigma_{1}+\sigma_{2}=2, \sigma_{1}<1\right), \\
O\left(T^{4-2 \sigma_{1}-2 \sigma_{2}}\right) & \left(\sigma_{1}+\sigma_{2}<2, \sigma_{1}<1\right) .\end{cases}
\end{aligned}
$$


Therefore, in the case $\sigma_{1}+\sigma_{2}>3 / 2$ and $\sigma_{2}>1 / 2$, we have

$$
\begin{aligned}
& \int_{T}^{2 T}\left|\zeta_{2}\left(s_{1}, s_{2}\right)\right|^{2} d t_{2} \\
&=\zeta_{2}^{[2]}\left(s_{1}, 2 \sigma_{2}\right) T+ \begin{cases}O(\log T) & \left(\sigma_{2}=1, \sigma_{1} \geq 1\right), \\
O\left(T^{2-2 \sigma_{2}}\right) & \left(\sigma_{2}<1, \sigma_{1} \geq 1\right), \\
O(\log T) & \left(\sigma_{1}+\sigma_{2}=2, \sigma_{1}<1\right), \\
O\left(T^{4-2 \sigma_{1}-2 \sigma_{2}}\right) & \left(\sigma_{1}+\sigma_{2}<2, \sigma_{1}<1\right) .\end{cases}
\end{aligned}
$$

In the case $\sigma_{1}>1, \sigma_{2}=1 / 2$, since

$$
a_{n}=n^{-\sigma_{2}} \sum_{m=1}^{n-1} m^{-s_{1}}=n^{-\sigma_{2}}\left(\zeta\left(s_{1}\right)+O\left(n^{-\sigma_{1}+1}\right)\right)
$$

by Lemma 2.2, we have

$$
\left|a_{n}\right|^{2}=n^{-1}\left|\zeta\left(s_{1}\right)+O\left(n^{-\sigma_{1}+1}\right)\right|^{2}=n^{-1}\left|\zeta\left(s_{1}\right)\right|^{2}+O\left(n^{-\sigma_{1}}\right) .
$$

Therefore, we obtain

$$
\int_{T}^{2 T}\left|\zeta_{2}\left(s_{1}, s_{2}\right)\right|^{2} d t_{2}=\left|\zeta\left(s_{1}\right)\right|^{2} T \log T+O(T)
$$

by (4.3). In the case $\sigma_{1}<1$ and $\sigma_{1}+\sigma_{2}=3 / 2$, since

$$
a_{n}=n^{-\sigma_{2}}\left(\frac{n^{-s_{1}+1}}{s_{1}-1}+O\left(n^{-\sigma_{1}}\right)+O(1)\right)
$$

by Lemma 2.2, we have

$$
\begin{aligned}
\left|a_{n}\right|^{2} & =n^{-2 \sigma_{2}}\left(\left|\frac{n^{-s_{1}+1}}{s_{1}-1}\right|^{2}+O\left(n^{-2 \sigma_{1}+1}\right)+O\left(n^{-\sigma_{1}+1}\right)\right) \\
& =\frac{n^{-1}}{\left|s_{1}-1\right|^{2}}+O\left(n^{-2}\right)+O\left(n^{-2+\sigma_{1}}\right) .
\end{aligned}
$$

Therefore, we obtain

$$
\int_{T}^{2 T}\left|\zeta_{2}\left(s_{1}, s_{2}\right)\right|^{2} d t_{2}=\frac{T \log T}{\left|s_{1}-1\right|^{2}}+O(T)
$$


by (4.3). In the case $\sigma_{1}=1$ and $\sigma_{2}=1 / 2$, since

$$
a_{n}=n^{-\sigma_{2}}\left(\zeta\left(s_{1}\right)-\frac{n^{-s_{1}+1}}{s_{1}-1}+O\left(n^{-\sigma_{1}}\right)\right)
$$

by Lemma 2.2, we have

$$
\sum_{n \leq T}\left|a_{n}\right|^{2}=\sum_{n \leq T}\left(n^{-1}\left|\zeta\left(s_{1}\right)-\frac{n^{-s_{1}+1}}{s_{1}-1}\right|^{2}+O\left(n^{-2}\right)\right)
$$

by Corollary 2.1. Since we have

$$
\begin{aligned}
& \sum_{n \leq T} n^{-1}\left|\zeta\left(s_{1}\right)-\frac{n^{-s_{1}+1}}{s_{1}-1}\right|^{2} \\
& \quad=\left(\left|\zeta\left(s_{1}\right)\right|^{2}+\left|s_{1}-1\right|^{-2}\right) \log T-2 \sum_{n \leq T} \Re\left(\overline{\zeta\left(s_{1}\right)} \frac{n^{-s_{1}}}{s_{1}-1}\right)+O(1) \\
& \quad=\left(\left|\zeta\left(s_{1}\right)\right|^{2}+\left|s_{1}-1\right|^{-2}\right) \log T+O(1)
\end{aligned}
$$

by Corollary 2.1, we have

$$
\sum_{n \leq T}\left|a_{n}\right|^{2}=\left(\left|\zeta\left(s_{1}\right)\right|^{2}+\left|s_{1}-1\right|^{-2}\right) \log T+O(1)
$$

Therefore, we obtain

$$
\int_{T}^{2 T}\left|\zeta_{2}\left(s_{1}, s_{2}\right)\right|^{2} d t_{2}=\left(\left|\zeta\left(s_{1}\right)\right|^{2}+\left|s_{1}-1\right|^{-2}\right) T \log T+O(T)
$$

by (4.3). By (4.4), (4.5), (4.6), and (4.7), we can obtain the theorem by the same argument as in the proof of Theorem 1.1.

Proof of Theorem 1.2 for $s_{1}=1$. We prove the theorem by the same argument as in the proof of Theorem 1.1.

Hereafter we use the same notation as in the preceding proof. Note that, in this case, we have $I_{2}=O\left(t_{2}^{-1} N^{1-\sigma_{2}} \log N\right)$ by using Lemma 4.1. Since $a_{n} \ll n^{-\sigma_{2}} \log n$, we obtain

$$
\sum_{n=2}^{N} n\left|a_{n}\right|^{2} \ll \sum_{n=2}^{N} n^{1-2 \sigma_{2}}(\log n)^{2} \ll \begin{cases}O\left((\log N)^{3}\right) & \left(\sigma_{2}=1\right), \\ O\left(N^{2-2 \sigma_{2}}(\log N)^{2}\right) & \left(\sigma_{2}<1\right)\end{cases}
$$


and

$$
I_{1} \ll \sum_{n=2}^{N}\left|a_{n}\right| \ll \begin{cases}(\log N)^{2} & \left(\sigma_{2}=1\right), \\ N^{1-\sigma_{2}} \log N & \left(\sigma_{2}<1\right) .\end{cases}
$$

Therefore, we have

$$
\int_{T}^{2 T}\left|I_{1}\right|^{2} d t_{2}=T \sum_{n=2}^{N}\left|a_{n}\right|^{2}+ \begin{cases}O\left((\log N)^{3}\right) & \left(\sigma_{2}=1\right) \\ O\left(N^{2-2 \sigma_{2}}(\log N)^{2}\right) & \left(\sigma_{2}<1\right)\end{cases}
$$

by Lemma 2.1 and

$$
\int_{T}^{2 T}\left|I_{1} I_{2}\right| d t_{2} \ll \begin{cases}O\left((\log N)^{3}\right) & \left(\sigma_{2}=1\right), \\ O\left(N^{2-2 \sigma_{2}}(\log N)^{2}\right) & \left(\sigma_{2}<1\right) .\end{cases}
$$

On the other hand, we have

$$
\int_{T}^{2 T}\left|I_{2}\right|^{2} d t_{2} \ll T^{-1} N^{2-2 \sigma_{2}}(\log N)^{2} .
$$

Therefore, by setting $N=[T]+1$, we obtain

$$
\int_{T}^{2 T}\left|\zeta_{2}\left(s_{1}, s_{2}\right)\right|^{2} d t_{2}=T \sum_{n \leq T}\left|a_{n}\right|^{2}+ \begin{cases}O\left((\log T)^{3}\right) & \left(\sigma_{2}=1\right) \\ O\left(T^{2-2 \sigma_{2}}(\log T)^{2}\right) & \left(\sigma_{2}<1\right)\end{cases}
$$

In the case $\sigma_{2}>1 / 2$, we have

$$
\begin{aligned}
& \int_{T}^{2 T}\left|\zeta_{2}\left(s_{1}, s_{2}\right)\right|^{2} d t_{2} \\
& \quad=\zeta_{2}^{[2]}\left(s_{1}, 2 \sigma_{2}\right) T+ \begin{cases}O\left((\log T)^{3}\right) & \left(\sigma_{2}=1\right), \\
O\left(T^{2-2 \sigma_{2}}(\log T)^{2}\right) & \left(\sigma_{2}<1\right) .\end{cases}
\end{aligned}
$$

In the case $\sigma_{2}=1 / 2$, since

$$
\left|a_{n}\right|^{2}=n^{-1}\left(\sum_{m=1}^{n-1} m^{-1}\right)^{2}=\frac{(\log n)^{2}}{n}+O\left(\frac{\log n}{n}\right)
$$

and

$$
\sum_{n=2}^{N} \frac{(\log n)^{2}}{n}=\int_{1}^{N} x^{-1}(\log x)^{2} d x+O(1)=\frac{(\log N)^{3}}{3}+O(1)
$$


hold, we have

$$
\int_{T}^{2 T}\left|\zeta_{2}\left(s_{1}, s_{2}\right)\right|^{2} d t_{2}=\frac{T(\log T)^{3}}{3}+O\left(T(\log T)^{2}\right)
$$

by (4.8). By (4.9) and (4.10), we can obtain the theorem by the same argument as in the proof of Theorem 1.1.

\section{§5. Proof of Theorem 1.3}

We divide the proof into four cases.

Proof of Theorem 1.3 for $\sigma_{2}>1$ and $\sigma_{1}+\sigma_{2}>2$. We set

$$
a_{k}=\left(\sum_{\substack{m \mid k \\ m<\sqrt{k}}} \frac{1}{m^{\sigma_{1}-\sigma_{2}}}\right) \frac{1}{k^{\sigma_{2}}}
$$

for $k \in \mathbb{N}$. We have

$$
\begin{aligned}
\zeta_{2}\left(s_{1}, s_{2}\right) & =\sum_{1 \leq m<n} \frac{1}{m^{\sigma_{1}} n^{\sigma_{2}}(m n)^{i t}} \\
& =\sum_{k \geq 2}\left(\sum_{\substack{m n=k \\
m<n}} \frac{1}{m^{\sigma_{1}} n^{\sigma_{2}}}\right) \frac{1}{k^{i t}} \\
& =\sum_{k \geq 2}\left(\sum_{\substack{m \mid k \\
m<\sqrt{k}}} \frac{1}{m^{\sigma_{1}-\sigma_{2}}}\right) \frac{1}{k^{\sigma_{2}+i t}} \\
& =\sum_{k \geq 2} a_{k} k^{-i t} .
\end{aligned}
$$

Since

$$
\sum_{k \geq 2} k\left|a_{k}\right|^{2}=\sum_{k \geq 2}^{\infty}\left(\sum_{\substack{m \mid k \\ m<\sqrt{k}}} \frac{1}{m^{\sigma_{1}-\sigma_{2}}}\right)^{2} \frac{1}{k^{2 \sigma_{2}-1}}
$$

converges by (2.3), we have

$$
I^{\square}(T)=\zeta_{2}^{\square}\left(\sigma_{1}, \sigma_{2}\right) T+O(1)
$$

by Lemma 2.1 . 
We use the following lemma in the cases $\sigma_{1}>1,1 / 2<\sigma_{2} \leq 1$ and $\sigma_{1} \leq 1$, $3 / 2<\sigma_{1}+\sigma_{2} \leq 2$.

Lemma 5.1. Let $\sigma_{1}+\sigma_{2}>1$, let $\sigma_{2}>0$, let $s_{1}=\sigma_{1}+i t$, and let $s_{2}=$ $\sigma_{2}+i t$. Then

$$
\zeta_{2}\left(s_{1}, s_{2}\right)=\sum_{n \leq t} n^{-s_{2}} \sum_{m=1}^{n-1} m^{-s_{1}}+ \begin{cases}O\left(t^{-\sigma_{2}}\right) & \left(\sigma_{1}>1\right) \\ O\left(t^{-\sigma_{2}+\epsilon}\right) & \left(\sigma_{1}=1\right) \\ O\left(t^{1-\sigma_{1}-\sigma_{2}}\right) & \left(\sigma_{1}<1\right)\end{cases}
$$

holds for $t \geq 2$, where the implied constants depend on $\sigma_{1}, \sigma_{2}$.

To prove Lemma 5.1, we use the following lemma and corollary.

Lemma 5.2 ([6, Lemma 2.2]). Let $s=\sigma+i t,|t|>1$. For $N>(1 / 4)|t|$, $m \geq 1$, and $\sigma>-2 m-1$, we have

$$
\begin{aligned}
\zeta(s)= & \sum_{n \leq N} \frac{1}{n^{s}}+\frac{N^{1-s}}{s-1}-\frac{N^{-s}}{2}+\sum_{k=1}^{2 m} \frac{B_{k+1}}{(k+1) !}(s)_{k} N^{-(s+k)} \\
& +O\left(|t|^{2 m+1} N^{-\sigma-2 m-1}\right)
\end{aligned}
$$

where the implied constant does not depend on $t$.

Corollary 5.1 ([6, Corollary 2.3]). Let $s=\sigma+i t,|t|>1$. For $N>$ $(1 / 4)|t|$ and $\sigma>-3$, we have

$$
\zeta(s)=\sum_{n \leq N} \frac{1}{n^{s}}+\frac{N^{1-s}}{s-1}-\frac{N^{-s}}{2}+\frac{s}{12} N^{-s-1}+O\left(|t|^{3} N^{-\sigma-3}\right),
$$

where the implied constant does not depend on $t$.

The following proof is similar to that in [6, Section 4.1].

Proof of Lemma 5.1. Let $l \in \mathbb{N}$ with $\sigma_{1}>-2 l$. We use (4.1) and (4.2). Hence, we obtain the analytic continuation of $\zeta_{2}\left(s_{1}, s_{2}\right)$ for $\sigma_{2}>0, \sigma_{1}>-2 l$, and $\sigma_{1}+\sigma_{2}>1$. Now, we set $s_{1}=\sigma_{1}+i t, s_{2}=\sigma_{2}+i t$ with $t \geq 1$ and $N=[t]$. 
Then we have

$$
\begin{aligned}
I_{1} & =\zeta\left(s_{1}\right)\left(\frac{N^{1-s_{2}}}{s_{2}-1}-\frac{N^{-s_{2}}}{2}+\frac{s_{2}}{12} N^{-s_{2}-1}+O\left(|t|^{3} N^{-\sigma_{2}-3}\right)\right) \\
& \ll\left|\zeta\left(s_{1}\right)\right| t^{-\sigma_{2}} \\
& \ll \begin{cases}t^{-\sigma_{2}} & \left(\sigma_{1}>1\right), \\
t^{-\sigma_{2}+\epsilon} & \left(\sigma_{1}=1\right), \\
t^{1-\sigma_{1}-\sigma_{2}} & \left(\sigma_{1}<1\right)\end{cases}
\end{aligned}
$$

for $\sigma_{2}>-3$ by Corollary 5.1. Similarly, we have

$$
\begin{aligned}
I_{2}= & \frac{1}{1-s_{1}}\left(\frac{N^{2-s_{1}-s_{2}}}{s_{1}+s_{2}-2}-\frac{1}{2} N^{1-s_{1}-s_{2}}+\frac{s_{1}+s_{2}-1}{12} N^{-s_{1}-s_{2}}\right. \\
& \left.+O\left(|t|^{3} N^{1-\sigma_{1}-\sigma_{2}-3}\right)\right) \\
& \ll t^{1-\sigma_{1}-\sigma_{2}}
\end{aligned}
$$

for $\sigma_{1}+\sigma_{2}>-2$. Since $\sigma_{1}+\sigma_{2}>1$, we have

$$
I_{j} \ll t^{1-\sigma_{1}-\sigma_{2}} \quad(j=3,4) .
$$

On the other hand, $R_{M, n}\left(s_{1}\right)=O\left(t^{M} n^{-\sigma_{1}-M}\right)$ for $\sigma_{1}>-M$ by Lemma 5.2. Hence, we have

$$
I_{5} \ll t^{M} \sum_{n>N} \frac{1}{n^{\sigma_{1}+\sigma_{2}+M}} \ll t^{1-\sigma_{1}-\sigma_{2}}
$$

This implies the lemma.

We prove Theorem 1.3 for $\sigma_{1}>1,1 / 2<\sigma_{2} \leq 1$ or $\sigma_{1} \leq 1,3 / 2<\sigma_{1}+$ $\sigma_{2} \leq 2$. First we consider the case $\sigma_{1}>\sigma_{2}$. In particular, this condition is satisfied when $\sigma_{1}>1$ and $1 / 2<\sigma_{2} \leq 1$.

Proof of Theorem 1.3 for $\sigma_{1}>\sigma_{2}$. If we set

$$
A\left(s_{1}, s_{2}\right)=\sum_{n \leq t} n^{-s_{2}} \sum_{m=1}^{n-1} m^{-s_{1}},
$$


then we have, say,

$$
\begin{aligned}
& \int_{2}^{T}\left|A\left(s_{1}, s_{2}\right)\right|^{2} d t \\
& =\int_{2}^{T}\left(\sum_{n_{1} \leq t} n_{1}^{-s_{2}} \sum_{m_{1}=1}^{n_{1}-1} m_{1}^{-s_{1}} \sum_{n_{2} \leq t} n_{2}^{-\overline{s_{2}}} \sum_{m_{2}=1}^{n_{2}-1} m_{2}^{-\overline{s_{1}}}\right) d t \\
& =\sum_{2 \leq n_{1} \leq T} \sum_{m_{1}=1}^{n_{1}-1} \sum_{2 \leq n_{2} \leq T} \sum_{m_{2}=1}^{n_{2}-1} n_{1}^{-\sigma_{2}} m_{1}^{-\sigma_{1}} n_{2}^{-\sigma_{2}} m_{2}^{-\sigma_{1}} \int_{M\left(n_{1}, n_{2}\right)}^{T}\left(\frac{m_{2} n_{2}}{m_{1} n_{1}}\right)^{i t} d t \\
& =\sum_{m_{1} n_{1}=m_{2} n_{2}} \sum_{\substack{1 \leq m_{1} \leq n_{1}-1 \\
2 \leq n_{1} \leq T}} \sum_{\substack{1 \leq m_{2} \leq n_{2}-1 \\
2 \leq n_{2} \leq T}} n_{1}^{-\sigma_{2}} m_{1}^{-\sigma_{1}} n_{2}^{-\sigma_{2}} m_{2}^{-\sigma_{1}}\left(T-M\left(n_{1}, n_{2}\right)\right) \\
& +\sum_{m_{1} n_{1} \neq m_{2} n_{2}} \sum_{\substack{1 \leq m_{1} \leq n_{1}-1 \\
2 \leq n_{1} \leq T}} \sum_{\substack{1 \leq m_{2} \leq n_{2}-1 \\
2 \leq n_{2} \leq T}} n_{1}^{-\sigma_{2}} m_{1}^{-\sigma_{1}} n_{2}^{-\sigma_{2}} m_{2}^{-\sigma_{1}} \\
& \times \frac{\exp \left(i T \log \left(\frac{m_{2} n_{2}}{m_{1} n_{1}}\right)\right)-\exp \left(i M\left(n_{1}, n_{2}\right) \log \left(\frac{m_{2} n_{2}}{m_{1} n_{1}}\right)\right)}{i \log \left(\frac{m_{2} n_{2}}{m_{1} n_{1}}\right)} \\
& =S_{1} T-S_{2}+S_{3} \text {, }
\end{aligned}
$$

where $M\left(n_{1}, n_{2}\right)=\max \left(n_{1}, n_{2}\right)$. First, we rewrite

$$
\begin{aligned}
S_{1}= & \sum_{2 \leq k \leq T}\left(\sum_{\substack{m n=k \\
m<n \leq T}} m^{-\sigma_{1}} n^{-\sigma_{2}}\right)^{2}+\sum_{T<k<T^{2}}\left(\sum_{\substack{m n=k \\
m<n \leq T}} m^{-\sigma_{1}} n^{-\sigma_{2}}\right)^{2} \\
= & \sum_{k=2}^{\infty}\left(\sum_{\substack{m n=k \\
m<n}} m^{-\sigma_{1}} n^{-\sigma_{2}}\right)^{2}-\sum_{k>T}\left(\sum_{\substack{m n=k \\
m<n}} m^{-\sigma_{1}} n^{-\sigma_{2}}\right)^{2} \\
& +\sum_{T<k<T^{2}}\left(\sum_{\substack{m n=k \\
m<n \leq T}} m^{-\sigma_{1}} n^{-\sigma_{2}}\right)^{2} .
\end{aligned}
$$

Since

$$
\begin{aligned}
\sum_{k>T}\left(\sum_{m n=k} m^{-\sigma_{1}} n^{-\sigma_{2}}\right)^{2} & =\sum_{k>T}\left(\sum_{m \mid k} m^{-\sigma_{1}} m^{\sigma_{2}} k^{-\sigma_{2}}\right)^{2} \\
& \ll \sum_{k>T} k^{-2 \sigma_{2}+\epsilon} \ll T^{1-2 \sigma_{2}+\epsilon},
\end{aligned}
$$


we have

$$
S_{1}=\zeta_{2}^{\square}\left(\sigma_{1}, \sigma_{2}\right)+O\left(T^{1-2 \sigma_{2}+\epsilon}\right) .
$$

Next, we rewrite, say,

$$
\begin{aligned}
S_{2} & \ll \sum_{m_{1} n_{1}=m_{2} n_{2}} \sum_{\substack{1 \leq m_{1} \leq n_{1}-1 \\
2 \leq n_{1} \leq T}} \sum_{\substack{1 \leq m_{2} \leq n_{2}-1 \\
2 \leq n_{2} \leq T}} n_{1}^{-\sigma_{2}} m_{1}^{-\sigma_{1}} n_{2}^{-\sigma_{2}} m_{2}^{-\sigma_{1}} O\left(n_{1}+n_{2}\right) \\
& \ll \sum_{2 \leq k<T^{2}}\left(\sum_{\substack{m n=k \\
1 \leq m<n \leq T}} m^{-\sigma_{1}} n^{1-\sigma_{2}}\right)\left(\sum_{\substack{m n=k \\
1 \leq m<n \leq T}} m^{-\sigma_{1}} n^{-\sigma_{2}}\right) \\
& \ll \sum_{2 \leq k \leq T}\left(\sum_{\substack{m n=k \\
1 \leq m<n \leq T}} m^{-\sigma_{1}} n^{1-\sigma_{2}}\right)\left(\sum_{\substack{m n=k \\
1 \leq m<n \leq T}} m^{-\sigma_{1}} n^{-\sigma_{2}}\right) \\
& +\sum_{T<k<T^{2}}\left(\sum_{\substack{m n=k \\
1 \leq m<n \leq T}} m^{-\sigma_{1}} n^{1-\sigma_{2}}\right)\left(\sum_{\substack{m n=k \\
1 \leq m<n \leq T}} m^{-\sigma_{1}} n^{-\sigma_{2}}\right) \\
= & A_{1}+A_{2} .
\end{aligned}
$$

Since we have

$$
\begin{aligned}
A_{1} & =\sum_{2 \leq k \leq T} k^{1-2 \sigma_{2}}\left(\sum_{\substack{m \mid k \\
m<\sqrt{k}}} m^{\sigma_{2}-\sigma_{1}-1}\right)\left(\sum_{\substack{m \mid k \\
m<\sqrt{k}}} m^{\sigma_{2}-\sigma_{1}}\right) \\
& \ll \sum_{2 \leq k \leq T} k^{1-2 \sigma_{2}+\epsilon} \ll T^{2-2 \sigma_{2}+\epsilon}
\end{aligned}
$$

and

$$
\begin{aligned}
A_{2} & \ll \sum_{T<k<T^{2}} k^{-2 \sigma_{1}}\left(\sum_{\substack{n \mid k \\
n \leq T}} n^{1+\sigma_{1}-\sigma_{2}}\right)\left(\sum_{\substack{n \mid k \\
n \leq T}} n^{\sigma_{1}-\sigma_{2}}\right) \\
& \ll T^{1+2 \sigma_{1}-2 \sigma_{2}+\epsilon} \sum_{T<k<T^{2}} k^{-2 \sigma_{1}} \ll T^{2-2 \sigma_{2}+\epsilon},
\end{aligned}
$$

we have $S_{2} \ll T^{2-2 \sigma_{2}+\epsilon}$. Next, we have, say,

$$
\begin{aligned}
S_{3}= & \sum_{m_{1} n_{1} \neq m_{2} n_{2}} \sum_{\substack{1 \leq m_{1} \leq n_{1}-1 \\
2 \leq n_{1} \leq T}} \sum_{\substack{1 \leq m_{2} \leq n_{2}-1 \\
2 \leq n_{2} \leq T}} n_{1}^{-\sigma_{2}} m_{1}^{-\sigma_{1}} n_{2}^{-\sigma_{2}} m_{2}^{-\sigma_{1}} \\
& \times \frac{\exp \left(i T \log \left(\frac{m_{2} n_{2}}{m_{1} n_{1}}\right)\right)-\exp \left(i M\left(n_{1}, n_{2}\right) \log \left(\frac{m_{2} n_{2}}{m_{1} n_{1}}\right)\right)}{i \log \left(\frac{m_{2} n_{2}}{m_{1} n_{1}}\right)}
\end{aligned}
$$




$$
\begin{aligned}
& \ll \sum_{m_{1} n_{1}<m_{2} n_{2}} \sum_{\substack{1 \leq m_{1} \leq n_{1}-1 \\
2 \leq n_{1} \leq T}} \sum_{\substack{1 \leq m_{2} \leq n_{2}-1 \\
2 \leq n_{2} \leq T}} n_{1}^{-\sigma_{2}} m_{1}^{-\sigma_{1}} n_{2}^{-\sigma_{2}} m_{2}^{-\sigma_{1}} \frac{1}{\log \left(\frac{m_{2} n_{2}}{m_{1} n_{1}}\right)} \\
& =\sum_{m_{1} n_{1}<m_{2} n_{2}<2 m_{1} n_{1}} \sum_{\substack{1 \leq m_{1} \leq n_{1}-1 \leq n_{1} \leq T \\
2}} \sum_{\substack{1 \leq m_{2} \leq n_{2}-1 \\
2 \leq n_{2} \leq T}} n_{1}^{-\sigma_{2}} m_{1}^{-\sigma_{1}} n_{2}^{-\sigma_{2}} m_{2}^{-\sigma_{1}} \frac{1}{\log \left(\frac{m_{2} n_{2}}{m_{1} n_{1}}\right)} \\
& \quad+\sum_{m_{2} n_{2} \geq 2 m_{1} n_{1}} \sum_{\substack{1 \leq m_{1} \leq n_{1}-1 \\
2 \leq n_{1} \leq T}} \sum_{\substack{1 \leq m_{2} \leq n_{2}-1 \\
2 \leq n_{2} \leq T}} n_{1}^{-\sigma_{2}} m_{1}^{-\sigma_{1}} n_{2}^{-\sigma_{2}} m_{2}^{-\sigma_{1}} \frac{1}{\log \left(\frac{m_{2} n_{2}}{m_{1} n_{1}}\right)} \\
& =B_{1}+B_{2} .
\end{aligned}
$$

We have $B_{2} \ll T^{2-2 \sigma_{2}}$ in the case $\sigma_{1}>1$. In the case $\sigma_{1} \leq 1$ we have

$$
B_{2} \ll \sum_{\substack{1 \leq m_{1} \leq n_{1}-1 \\ 2 \leq n_{1} \leq T}} \sum_{\substack{1 \leq m_{2} \leq n_{2}-1 \\ 2 \leq n_{2} \leq T}} n_{1}^{-\sigma_{2}} m_{1}^{-\sigma_{1}} n_{2}^{-\sigma_{2}} m_{2}^{-\sigma_{1}} \ll T^{4-2 \sigma_{1}-2 \sigma_{2}+\epsilon} .
$$

Hence, we have

$$
B_{2} \ll \begin{cases}T^{2-2 \sigma_{2}+\epsilon} & \left(\sigma_{1}>1\right) \\ T^{4-2 \sigma_{1}-2 \sigma_{2}+\epsilon} & \left(\sigma_{1} \leq 1\right) .\end{cases}
$$

Next we evaluate $B_{1}$. In the case $\sigma_{1}>1$ we have

$$
\begin{aligned}
& B_{1} \ll \sum_{r \leq T^{2}} \sum_{\substack{m_{2} n_{2}-r=m_{1} \\
r<m_{2} n_{2}}} \sum_{1 \leq m_{1}<n_{1} \leq T} \sum_{1 \leq m_{2}<n_{2} \leq T} n_{1}^{-\sigma_{2}} m_{1}^{-\sigma_{1}} n_{2}^{-\sigma_{2}} m_{2}^{-\sigma_{1}} \frac{m_{1} n_{1}}{r} \\
& \ll \sum_{r \leq T^{2}} \sum_{2 \leq n_{1} \leq T} \sum_{1 \leq m_{2}<n_{2} \leq T} \sum_{\substack{n_{1} \mid\left(m_{2} n_{2}-r\right) \\
r<m_{2} n_{2}}} n_{1}^{1-\sigma_{2}} n_{2}^{-\sigma_{2}} m_{2}^{-\sigma_{1}} \frac{1}{r} \\
& \ll \sum_{r \leq T^{2}} \sum_{2 \leq n_{1} \leq T} \sum_{1 \leq m_{2}<n_{2} \leq T} T^{1-\sigma_{2}+\epsilon} n_{2}^{-\sigma_{2}} m_{2}^{-\sigma_{1}} \frac{1}{r} \ll T^{2-2 \sigma_{2}+\epsilon} .
\end{aligned}
$$

If $\sigma_{1} \leq 1$ we have

$$
\begin{aligned}
B_{1} \ll & \sum_{r \leq T^{2}} \sum_{1 \leq m_{1}<n_{1} \leq T} \sum_{1 \leq m_{2}<n_{2} \leq T} \sum_{\substack{m_{2} n_{2}=m_{1} n_{1}+r \\
r<m_{2} n_{2}}} n_{1}^{-\sigma_{2}} m_{1}^{-\sigma_{1}} n_{2}^{-\sigma_{2}} m_{2}^{-\sigma_{1}} \\
& \times \frac{m_{1} n_{1}}{r} \\
(5.2) \ll & \sum_{r \leq T^{2}} \sum_{1 \leq m_{1}<n_{1} \leq T} \sum_{1 \leq m_{2}<n_{2} \leq T} \sum_{m_{2} n_{2}=m_{1} n_{1}+r} n_{1}^{-\sigma_{2}} m_{1}^{-\sigma_{1}} n_{2}^{-\sigma_{2}} m_{2}^{-\sigma_{1}}
\end{aligned}
$$




$$
\begin{aligned}
& \times \frac{m_{1} n_{1}}{r} \frac{m_{2} n_{2}}{m_{1} n_{1}} \\
\ll & \sum_{r \leq T^{2}} \sum_{1 \leq m_{1}<n_{1} \leq T} n_{1}^{-\sigma_{2}} m_{1}^{-\sigma_{1}} \frac{T^{2-\sigma_{1}-\sigma_{2}+\epsilon}}{r} \ll T^{4-2 \sigma_{1}-2 \sigma_{2}+\epsilon},
\end{aligned}
$$

since we are in the case $\sigma_{2} \leq \sigma_{1} \leq 1$. Hence, we have

$$
B_{1} \ll \begin{cases}T^{2-2 \sigma_{2}+\epsilon} & \left(\sigma_{1}>1\right) \\ T^{4-2 \sigma_{1}-2 \sigma_{2}+\epsilon} & \left(\sigma_{1} \leq 1\right)\end{cases}
$$

This implies that

$$
S_{3} \ll \begin{cases}T^{2-2 \sigma_{2}+\epsilon} & \left(\sigma_{1}>1\right) \\ T^{4-2 \sigma_{1}-2 \sigma_{2}+\epsilon} & \left(\sigma_{1} \leq 1\right)\end{cases}
$$

Therefore, we have

$$
\int_{2}^{T}\left|A\left(s_{1}, s_{2}\right)\right|^{2} d t=\zeta_{2}^{\square}\left(\sigma_{1}, \sigma_{2}\right) T+ \begin{cases}O\left(T^{2-2 \sigma_{2}+\epsilon}\right) & \left(\sigma_{1}>1\right), \\ O\left(T^{4-2 \sigma_{1}-2 \sigma_{2}+\epsilon}\right) & \left(\sigma_{1} \leq 1\right) .\end{cases}
$$

Now, if we set

$$
\lambda= \begin{cases}-\sigma_{2} & \left(\sigma_{1}>1\right) \\ -\sigma_{2}+\epsilon & \left(\sigma_{1}=1\right) \\ 1-\sigma_{1}-\sigma_{2} & \left(\sigma_{1}<1\right)\end{cases}
$$

then we have

$$
\begin{aligned}
\int_{2}^{T}\left|\zeta\left(s_{1}, s_{2}\right)\right|^{2} d t & =\int_{2}^{T}\left|A\left(s_{1}, s_{2}\right)+O\left(t^{\lambda}\right)\right|^{2} d t \\
& =\int_{2}^{T}\left|A\left(s_{1}, s_{2}\right)\right|^{2} d t+O\left(\int_{2}^{T}\left|A\left(s_{1}, s_{2}\right) t^{\lambda}\right| d t\right)+O(1) .
\end{aligned}
$$

By the Cauchy-Schwarz inequality, we have

$$
\begin{aligned}
\int_{2}^{T}\left|A\left(s_{1}, s_{2}\right) t^{\lambda}\right| d t & \ll\left(\int_{2}^{T}\left|A\left(s_{1}, s_{2}\right)\right|^{2} d t\right)^{1 / 2}\left(\int_{2}^{T} t^{2 \lambda} d t\right)^{1 / 2} \\
& \ll T^{1 / 2}
\end{aligned}
$$

This implies the theorems. 
Next, we consider the case $\sigma_{1} \leq \sigma_{2}$.

Proof of Theorem 1.3 for $\sigma_{1} \leq \sigma_{2}$. Hereafter we use the same notation as in the preceding proof. First we evaluate $S_{1}$. Since

$$
\begin{aligned}
& \sum_{k>T}\left(\sum_{\substack{m n=k \\
m<n}} m^{-\sigma_{1}} n^{-\sigma_{2}}\right)^{2} \\
& =\sum_{k>T}\left(\sum_{\substack{m \mid k \\
m<\sqrt{k}}} m^{-\sigma_{1}} m^{\sigma_{2}} k^{-\sigma_{2}}\right)^{2} \\
& \ll \sum_{k>T} k^{-2 \sigma_{2}}\left(k^{\frac{1}{2}\left(\sigma_{2}-\sigma_{1}\right)+\epsilon}\right)^{2} \\
& \ll \sum_{k>T} k^{-\sigma_{1}-\sigma_{2}+\epsilon} \ll T^{1-\sigma_{1}-\sigma_{2}+\epsilon},
\end{aligned}
$$

we have

$$
S_{1}=\zeta_{2}^{\square}\left(\sigma_{1}, \sigma_{2}\right)+O\left(T^{1-\sigma_{1}-\sigma_{2}+\epsilon}\right) .
$$

Next we evaluate $S_{2}$. Since

$$
\begin{aligned}
\sum_{\substack{m n=k \\
m<n}} m^{-\sigma_{1}} n^{-\sigma_{2}} & =\sum_{\substack{m \mid k \\
m<\sqrt{k}}} m^{\sigma_{2}-\sigma_{1}} k^{-\sigma_{2}} \ll k^{-\frac{1}{2}\left(\sigma_{1}+\sigma_{2}\right)+\epsilon}, \\
\sum_{\substack{m n=k \\
m<n}} m^{-\sigma_{1}} n^{1-\sigma_{2}} & =\sum_{\substack{m \mid k \\
m<\sqrt{k}}} k^{1-\sigma_{2}} m^{\sigma_{2}-\sigma_{1}-1} \\
& \ll \begin{cases}k^{1-\sigma_{2}+\epsilon} & \left(\sigma_{2}-\sigma_{1}-1 \leq 0\right), \\
k^{\frac{1}{2}\left(1-\sigma_{1}-\sigma_{2}\right)+\epsilon} & \left(\sigma_{2}-\sigma_{1}-1>0\right)\end{cases}
\end{aligned}
$$

hold, we have

$$
\begin{aligned}
A_{1} & \ll \begin{cases}\sum_{2 \leq k \leq T} k^{-\frac{1}{2}\left(\sigma_{1}+\sigma_{2}\right)+\epsilon} k^{1-\sigma_{2}+\epsilon} & \left(\sigma_{2}-\sigma_{1}-1 \leq 0\right), \\
\sum_{2 \leq k \leq T} k^{-\frac{1}{2}\left(\sigma_{1}+\sigma_{2}\right)+\epsilon} k^{\frac{1}{2}\left(1-\sigma_{1}-\sigma_{2}\right)+\epsilon} & \left(\sigma_{2}-\sigma_{1}-1>0\right),\end{cases} \\
& = \begin{cases}\sum_{2 \leq k \leq T} k^{1-\frac{1}{2} \sigma_{1}-\frac{3}{2} \sigma_{2}+\epsilon} & \left(\sigma_{2}-\sigma_{1}-1 \leq 0\right), \\
\sum_{2 \leq k \leq T} k^{\frac{1}{2}-\sigma_{1}-\sigma_{2}+\epsilon} & \left(\sigma_{2}-\sigma_{1}-1>0\right) .\end{cases}
\end{aligned}
$$


We note that $1-(1 / 2) \sigma_{1}-(3 / 2) \sigma_{2}<-1$ is equivalent to $\sigma_{2}>-(1 / 3) \sigma_{1}+$ $(4 / 3)$. Hence, we have

$$
A_{1} \ll \begin{cases}T^{2-\frac{1}{2} \sigma_{1}-\frac{3}{2} \sigma_{2}+\epsilon} & \left(\sigma_{2}-\sigma_{1}-1 \leq 0 \text { and } \sigma_{2} \leq-\frac{1}{3} \sigma_{1}+\frac{4}{3}\right) \\ 1 & \text { (otherwise) }\end{cases}
$$

because $\sigma_{1}+\sigma_{2}>3 / 2$. Similarly, we have

$$
\begin{aligned}
A_{2} & \ll \begin{cases}\sum_{T<k<T^{2}} k^{1-\frac{1}{2} \sigma_{1}-\frac{3}{2} \sigma_{2}+\epsilon} & \left(\sigma_{2}-\sigma_{1}-1 \leq 0\right), \\
\sum_{T<k<T^{2}} k^{\frac{1}{2}-\sigma_{1}-\sigma_{2}+\epsilon} & \left(\sigma_{2}-\sigma_{1}-1>0\right),\end{cases} \\
& \ll \begin{cases}T^{4-\sigma_{1}-3 \sigma_{2}+\epsilon} & \left(\sigma_{2}-\sigma_{1}-1 \leq 0 \text { and } \sigma_{2} \leq-\frac{1}{3} \sigma_{1}+\frac{4}{3}\right), \\
1 & \text { (otherwise) } .\end{cases}
\end{aligned}
$$

Therefore, we have

$$
S_{2} \ll \begin{cases}T^{4-\sigma_{1}-3 \sigma_{2}+\epsilon} & \left(\sigma_{2}-\sigma_{1}-1 \leq 0 \text { and } \sigma_{2} \leq-\frac{1}{3} \sigma_{1}+\frac{4}{3}\right), \\ 1 & \text { (otherwise). }\end{cases}
$$

Next we evaluate $S_{3}$. Since estimation (5.1) remains also valid in this case, we have $B_{2} \ll T^{4-2 \sigma_{1}-2 \sigma_{2}+\epsilon}$. Since we have

$$
\begin{aligned}
\sum_{1 \leq m_{2}<n_{2} \leq T} \sum_{m_{2} n_{2}=m_{1} n_{1}+r} n_{2}^{1-\sigma_{2}} m_{2}^{1-\sigma_{1}} & \ll\left(\frac{m_{1} n_{1}+r}{T}\right)^{1-\sigma_{2}} T^{1-\sigma_{1}+\epsilon} \\
& \ll T^{2-\sigma_{1}-\sigma_{2}+\epsilon}
\end{aligned}
$$

for $\sigma_{1} \leq 1, \sigma_{2}>1,0 \neq m_{1} n_{1}+r \ll T^{2}$, estimation (5.2) also remains valid in this case. Therefore, we have $S_{3} \ll T^{4-2 \sigma_{1}-2 \sigma_{2}+\epsilon}$. Since $4-2 \sigma_{1}-2 \sigma_{2}-$ $\left(4-\sigma_{1}-3 \sigma_{2}\right)=\sigma_{2}-\sigma_{1} \geq 0$, we have

$$
\int_{2}^{T}\left|A\left(s_{1}, s_{2}\right)\right|^{2} d t=\zeta_{2}^{\square}\left(\sigma_{1}, \sigma_{2}\right) T+O\left(T^{4-2 \sigma_{1}-2 \sigma_{2}+\epsilon}\right) .
$$

By the same argument as in the case $\sigma_{1}>\sigma_{2}$, we obtain the theorem.

Proof of Theorem 1.3 for $\sigma_{1}>1$ and $\sigma_{2}=1 / 2$. By [11, Theorem 2.2] we have

$$
\int_{2}^{T}|\zeta(1 / 2+i t)|^{2}\left|\zeta\left(\sigma_{1}+i t\right)\right|^{2} d t \sim \frac{\zeta\left(2 \sigma_{1}\right) \zeta\left(\sigma_{1}+1 / 2\right)^{2}}{\zeta\left(2 \sigma_{1}+1\right)} T \log T
$$


By (1.3) and the Cauchy-Schwarz inequality, we have

$$
I^{\square}(T) \sim \int_{2}^{T}|\zeta(1 / 2+i t)|^{2}\left|\zeta\left(\sigma_{1}+i t\right)\right|^{2} d t .
$$

This completes the proof.

Acknowledgments. We would like to thank Kohji Matsumoto for his useful advice. We are very grateful to the anonymous referees for their helpful suggestions and remarks, which significantly improved this article.

\section{REFERENCES}

[1] S. Akiyama, S. Egami, and Y. Tanigawa, Analytic continuation of multiple zetafunctions and their values at non-positive integers, Acta Arith. 98 (2001), 107-116. MR 1831604. DOI 10.4064/aa98-2-1.

[2] F. V. Atkinson, The mean-value of the Riemann zeta function, Acta Math. 81 (1949), 353-376. MR 0031963.

[3] H. M. Edwards, Riemann's Zeta Function, Pure Appl. Math. 58, Academic Press, New York, 1974. MR 0466039.

[4] M. E. Hoffman, Multiple harmonic series, Pacific J. Math. 152 (1992), 275-290. MR 1141796.

[5] A. Ivić, The Riemann Zeta-Function: The Theory of the Riemann Zeta-Function with Applications, Wiley-Intersci. Publ., Wiley, New York, 1985. MR 0792089.

[6] I. Kiuchi and Y. Tanigawa, Bounds for double zeta-functions, Ann. Sc. Norm. Super. Pisa Cl. Sci. (5) 5 (2006), 445-464. MR 2297719.

[7] I. Kiuchi, Y. Tanigawa, and W. Zhai, Analytic properties of double zeta-functions, Indag. Math. (N.S.) 21 (2011), 16-29. MR 2832479. DOI 10.1016/j.indag.2010.12.001.

[8] K. Matsumoto, "On the analytic continuation of various multiple zeta-functions" in Number Theory for the Millennium, II (Urbana, 2000), A. K. Peters, Natick, Mass., 2002, 417-440. MR 1956262.

[9] , Functional equations for double zeta-functions, Math. Proc. Cambridge Philos. Soc. 136 (2004), 1-7. MR 2034011. DOI 10.1017/S0305004103007035.

[10] K. Matsumoto and H. Tsumura, Mean value theorems for the double zeta-function, J. Math. Soc. Japan 67 (2015), 383-406. MR 3304026.

[11] S. Shimomura, Fourth moment of the Riemann zeta-function with a shift along the real line, Tokyo J. Math. 36 (2013), 355-377. MR 3161563. DOI 10.3836/tjm/1391177976.

[12] E. C. Titchmarsh, The Theory of Functions, reprint of the 2nd ed., Oxford University Press, Oxford, 1958. MR 3155290.

[13] - The Theory of the Riemann Zeta-Function, 2nd ed., Oxford University Press, New York, 1986. MR 0882550.

[14] D. Zagier, "Values of zeta-functions and their applications" in First European Congress of Mathematics, Vol. II (Paris, 1992), Progr. Math. 120, Birkhäuser, Basel, 1994, 497-512. MR 1341859. 
[15] J. Q. Zhao, Analytic continuation of multiple zeta function, Proc. Amer. Math. Soc. 128 (2000), 1275-1283. MR 1670846. DOI 10.1090/S0002-9939-99-05398-8.

Soichi Ikeda

Graduate School of Mathematics

Nagoya University

Furocho, Chikusaku

Nagoya 464-8602

Japan

m10004u@math.nagoya-u.ac.jp

Kaneaki Matsuoka

Graduate School of Mathematics

Nagoya University

Furocho, Chikusaku

Nagoya 464-8602

Japan

m10041v@math.nagoya-u.ac.jp

Yoshikazu Nagata

Graduate School of Mathematics

Nagoya University

Furocho, Chikusaku

Nagoya 464-8602

Japan

m10035y@math. nagoya-u.ac.jp 